\title{
CARBON NANOTUBE MEMBRANE PROBES:IMMUNO-LABELING BY LM, AFM, TEM, \& FESEM
}

\author{
B.Panessa-Warren*, S.S.Wong,** B.Ghebrehiwet, ${ }^{* * *}$ G.T.Tortora ****and J.B.Warren*
}

Instrumentation Division, Brookhaven National Laboratory,Upton, NY11973

**Chemistry Dept., State University of NY, Stony Brook, NY11794 and Materials Science

Dept., Brookhaven National Laboratory, Upton, NY 11973

***Dept. of Medicine, State University of NY, Stony Brook, NY11794

****Clin.Microbiology Lab., University Hospital, SUNY, Stony Brook, NY

Single walled carbon nanotubes (SWNTs) with their carbon lattice structure have unique mechanical, and structure-dependent electronic properties[1,3]. By heating and sonicating SWNTs in $\mathrm{H}_{2} \mathrm{SO}_{4}$ and $\mathrm{H}_{2} \mathrm{O}_{2}$, nanotubes and nanoropes were modified[2], and covalently functionalized $[1,3]$ with monoclonal antibodies and purified membrane proteins. This investigation demonstrates the development and application of carbon nanoloops [4], functionalized with (a) gClq-R specific monoclonal antibodies and (b) gClq receptor protein (the latter is the cellular receptor for InlB-mediated entry of Listeria monocytogenes into mammalian cells). These antibody-probes were incubated with human tissue culture cells (Caco-2 cells), to allow surface immuno-attachment. Immuno-reacted cell monolayers were then incubated with Listeria monocytogenes bacteria, or Clostridium difficile endospores, to see if blocking the receptor sites on the epithelial membrane prevented entry of these two pathogens into the host cells.

SWNTs were cut [2] and then sonicated with 1-ethyl-3(3-dimetylaminopropyl) carbodiimide hydrochloride (EDC) [1,3]. PBS buffer-washed nanoloops were incubated with monoclonal antibody or gClq receptor protein in PBS with agitation. Carbon nanostructures were imaged and their dimensions measured using a Digital Instruments Nanoscope IIIa Multimode instrument (AFM). Aliquots of EDC-, and antibody-treated nanoloops were deposited on mica and imaged using tapping mode in air. Monolayers of $\mathrm{Caco} 2$ cells (Diagnostic Hybrids, Athens, Ohio), were pre-incubated with functionalized nanoloops. Aliquots of Listeria monocytogenes, or Clostridium difficile endospore preparations were added to each vial and the preparations incubated. Caco- 2 cell monolayers were fixed in $3 \%$ glutaraldehyde in $0.1 \mathrm{M}$ cacodylate buffer $(\mathrm{pH} 7.2)$ with sucrose, followed by post fixation in $1 \%$ aqueous $\mathrm{OsO}_{4}$ with sucrose, and prepared for thin section TEM examination, or for FESEM by critical point drying, Pt coating (3-5nm), and examination at 5 or $10 \mathrm{kV}$.

AFM (Fig.1a) and negatively stained TEM (Fig. 1b) images of functionalized nanoloops showed varying sizes of elliptical and spherical rings, with protein around the outer skeleton of each nanoloop and within the central hole. Antibody functionalized nanoloops did not attach to the Caco- 2 cell membrane surfaces of non-bacteria-incubated controls. Those vials incubated with bacteria showed extensive clusters of functionalized nanoloops on the epithelial cell surface, similar to "capping" or "patching". Where the antibodyfunctionalized nanoloops had attached to the plasmalemma, no Listeria bacteria were found entering the cells. This suggested that the gClq receptor site had been blocked by the 
antibody, and therefore blocked entry of the Listeria into the cell at that locus. Similarly, C.difficile endospores that normally showed rapid, homogeneous attachment to Caco 2 cell microvilli, did not attach to cells showing functionalized nanoloopantibody reactivity. By AFM, we found nanotube loops varying from small spherical, to larger elliptical, structures following EDC treatment. Biologically, the functionalized nanoloop antibody probes attached to receptor sites on the host cell membranes, which were not removed by successive washes in PBS, water, or acetone. These functionalized biological membrane probes permitted dynamic experiments with living cells and microorganisms, which could be visualized by conventional LM, TEM, AFM and FESEM, making this a versatile, and easy to use, specific immuno-probe.

\section{REFERENCES}

[1]S.S.Wong et al., Nature, 394 (1998)52.

[2]J.Liu et al., Science, 280(1998)1253.

[3]S.Banerjee \& S.S.Wong, Nano Letters 2(1)(2002)49.

[4]M.Sano et al., Science, 293 (2001)1299.

[5]This work was partially supported by grant DEAC02-98CH10886.

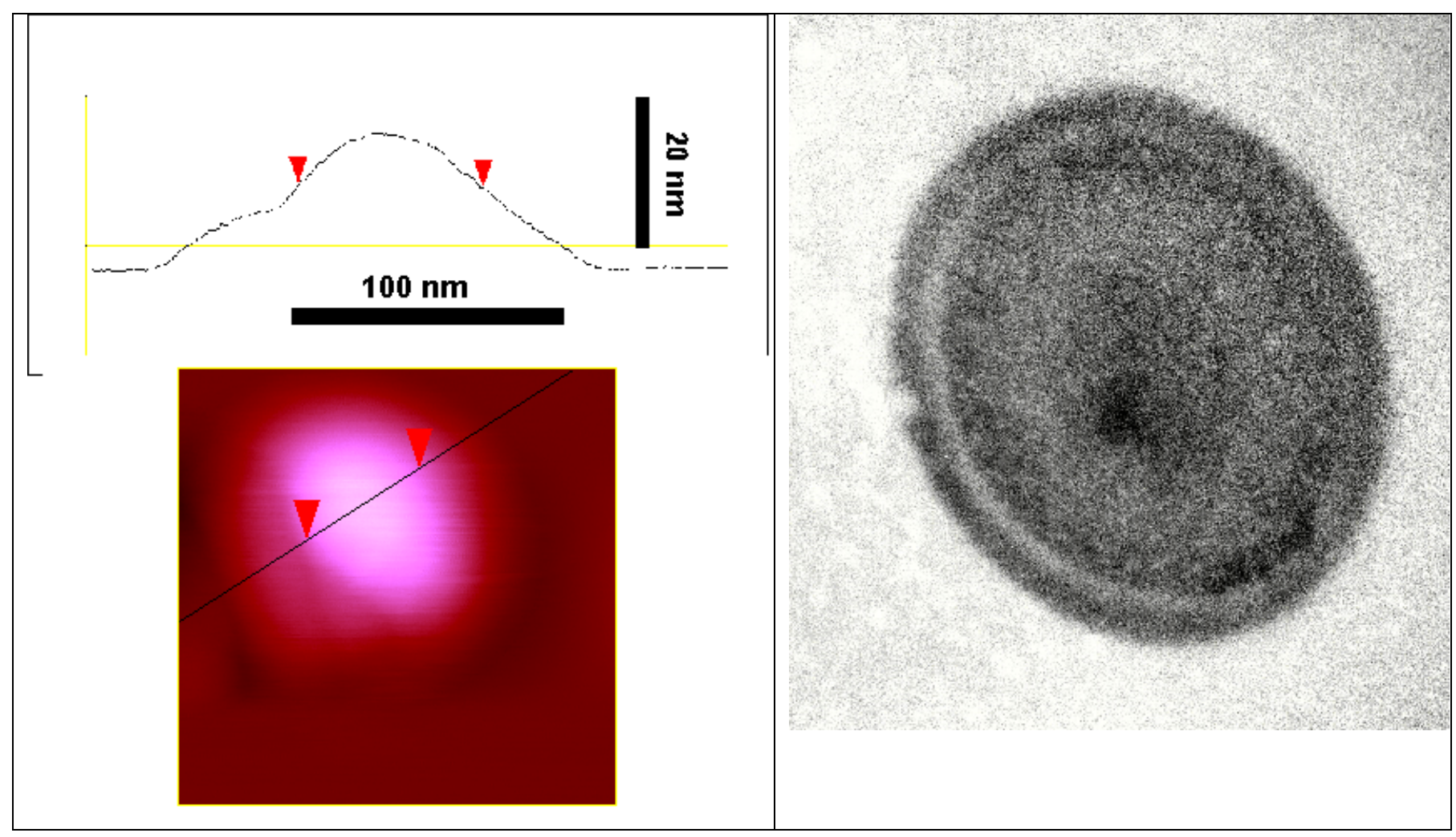

Fig. 1a.

Fig. 1b.

Fig. 1. (a). Tapping Mode AFM height image (with cross -section above) and (b). TEM image (100 nm x $100 \mathrm{~nm})$ of an identically-prepared antibodyfunctionalized nanoloop. 